\title{
Expression of Magnaporthe oryzae genes encoding cysteine-rich proteins secreted during nitrogen starvation and interaction with its host, Oryza sativa
}

\author{
J. Yang*, M.L. Liang*, J.L. Yan, Y.Q. Yang, L. Liu, C. Liu, L.J. Yang and C.Y. Li \\ Key Laboratory for Agricultural Biodiversity and Pest Management of China Education \\ Ministry, Yunnan Agricultural University, Heilongtan, Kunming, Yunnan, China \\ *These authors equally contributed to this study. \\ Corresponding author: C.Y. Li \\ E-mail: licheng-yun@163.com \\ Genet. Mol. Res. 14 (4): 17099-17108 (2015) \\ Received August 15, 2015 \\ Accepted October 10, 2015 \\ Published December 16, 2015 \\ DOI http://dx.doi.org/10.4238/2015.December.16.10
}

\begin{abstract}
Previous studies have shown that the blast fungus, Magnaporthe oryzae, may experience nitrogen starvation during infection of its plant host (rice,Oryza sativa). Here, we studied the expression of seven genes encoding cysteine-rich proteins with $\mathrm{N}$-terminal signal peptides during nitrogen limitation and throughout the infection process. Some genes were upregulated to a greater extent in weak pathogenic strains than in strong pathogenic strains when they were cultured in complete media, and the expression of some genes was higher in both weak and strong pathogenic strains cultured in 1/10-N and nitrogen starvation media. Furthermore, the expression of these genes was upregulated to different extents in the early stages of $M$. oryzae infection. These data demonstrate that the genes of interest are highly expressed in weak and strong pathogenic strains cultured under nitrogen limitation and at the early stage of the infection process. This indicates that cysteine-rich secreted proteins in the blast fungus might be involved in establishing disease in
\end{abstract}


the host and that they are sensitive to nitrogen levels. Thus, their role in sensing nitrogen availability within the host is implied, which provides a basis for further functional identification of these genes and their products during plant infection.

Key words: Rice blast fungus; Rice; Small secreted proteins; Interaction; Nitrogen resource

\section{INTRODUCTION}

Fungi utilize a variety of nitrogen sources from host tissues in order to infect and colonize their hosts. However, the precise mechanism of how these organisms utilize the available source(s) of host nitrogen is unknown. Presently, it is understood that the nutritional status of the host involves the induction of specific genes (Snoeijers et al., 2000). Furthermore, nitrogen metabolism of fungi directly influences the extent of fungal colonization of host tissue (Donofrio et al., 2006). Pathogens utilize nitrogen sources depending on the host tissues being colonized. There are obvious differences between a root pathogen and a leaf pathogen (Snoeijers et al., 2000). Necrotrophic pathogens effectively utilize more of the available nitrogen present in the host tissue than do biotrophs, because biotrophic fungi only utilize nitrogen sources that are available in the apoplast and the haustorial matrix of the host (Snoeijers et al., 2000).

Research on microbial nitrogen metabolism and regulation has previously been performed in model microorganisms such as the enteric bacteria Escherichia coli and Salmonella spp (Magasanik et al., 1996), and in filamentous fungi such as Aspergillus nidulans and Neurospora crassa (Marzluf et al., 1997). The response of $A$. nidulans and $N$. crassa to the nitrogen source is regulated by transcription factors (TFs). Activation of such TFs depends upon the nitrogen source and quantum (Marzluf et al., 1997). It has also been reported that nitrogen limitation is an important 'signal' that triggers the expression of virulence genes in plant pathogens (Snoeijers et al., 2000). Nitrogen limitation can activate, or induce the expression of TFs that subsequently upregulate nitrogen metabolism. All of these TFs contain zinc-finger domains and they bind to GATA promoter sequences, which triggers the transcription of genes involved in nitrogen metabolism. Several studies have demonstrated that the Mpg1 gene of Magnaporthe oryzae and the avirulence gene Avr9 of the tomato pathogen Cladosporium fulvum (Talbot et al., 1993; Van den Ackerveken et al., 1994) are strongly upregulated under nitrogen limitation.

Avr9 encodes a small, 63-amino acid protein with six cysteine residues. When the plant pathogen $C$. fulvum was cultured under conditions of nitrogen starvation, upregulation of Avr9 resulted in a hypersensitive response in the host carrying the corresponding resistance gene (Van den Ackerveken et al., 1994). Previous research has also shown that NPR1 and NPR2 regulate nitrogen metabolism and are also indispensable for blast fungus pathogenesis (Lau et al., 1996). Several studies have demonstrated that small, cysteine-rich proteins play a role in the specificity and pathogenicity of fungal pathogens (Templeton et al., 1994). For example, Avr9 (Snoeijerset al.,1999), Avr4 (Joosten et al.,1994), and a putative avirulence gene product from Rhynchosporium secalis (Wevelsiep et al., 1991) have low molecular weights and no obvious sequence homology to each other, or to other known proteins. They contain 6-10 cysteine residues in their amino acid sequence. Furthermore, some elicitins with molecular weights of $10 \mathrm{kDa}$ characterized from Phytophthora spp have also been shown to cause necrosis of host plants, to trigger Hazard Risk (HR), and to activate the expression of pathogenicity-related genes when used to treat the non-host tobacco (Ricci et al., 
1989; Nespoulous et al., 1992; Kamoun et al., 1993; Pernollet et al., 1994).

In the present study, we used predictive software to identify genes encoding putative secretory proteins within the genome sequence of $M$. oryzae. We performed similarity searches in the NCBI database to obtain candidate genes encoding small secretory proteins in rich cysteine residues and without homology to any known genes. Primer pairs were then designed based on the sequences of candidate genes. Subsequently, we analyzed the expression levels of the selected genes in the two blast strains cultured in complete media, 1/10-N media, nitrogen starvation media, and at different stages of the infection process. We identified seven genes encoding small cysteine-rich secretory proteins that are sensitive to their nitrogen source. The data obtained provide important information on the study of pathogen growth and development following adaptation to nitrogen limitation.

\section{MATERIAL AND METHODS}

\section{Rice and $M$. oryzae strains}

The rice cultivar Lijiangxintuanheigu (LTH) was used, which is susceptible to the majority of isolates that cause rice blast. The strains of rice blast fungus used were Y98-16, Y99-63, 95-23-4a, and 94-64-1b. We previously performed a pathogenicity test for the four strains; Y98-16 and 95-23-4a were strong pathogenic strains, Y99-63and 94-64-1b were weak pathogenic strains (data not shown)

\section{Sequence characterization}

Putative small proteins sequences $(<100$ aa) in the rice blast fungus $(M$. oryzae) genome database (version 1.0) were accessed at http://www.broad.mit.edu/cgi-bin/annotation/magnaporthe/download_license.cgi.

The secretory proteins of $M$. oryzae were predicted using internet-based software such as SignalP4.0, TargetP V1.1, Big-PI predictor, and TMHMM v2.0 (http://www.cbs.dtu.dk/services/), and the determinant standard of "typical" secretory proteins conformed to the following four standards; standard four is $L=-918.235-123.455^{\star}$ (Mean S score) $+1983.44^{*}$ (HMM score) and $L>0$ for predicting proteins with a signal peptide; standard two is no transmembrane helix; standard three is the use of TargetP to remove proteins that are predicted to be targeted to mitochondria; and standard four is no GPI-anchor site (Lee et al., 2003).

Bioinformatic analysis of the gene MoSDT1 and its predicted protein was performed using the software ExPASY proteomics server to analyze molecular weight and domain (http://www. expasy.ch/tools/pi_tool.html), MultiLoc/TargetLoc (http://www-bs.informatik.uni-tuebingen.de/Services/MultiLoc) was used to predict the subcellular localization.

\section{Nitrogen limitation experiments}

For nitrogen limitation experiments, $M$. oryzae mycelia were grown on potato dextrose agar at $28^{\circ} \mathrm{C}$, and a piece of mycelia was inoculated into complete media $\left\{6 \mathrm{~g} \mathrm{NaNO}_{3}, 0.52 \mathrm{~g} \mathrm{KCl}\right.$, $0.52 \mathrm{~g} \mathrm{MgSO}_{4} \cdot 7 \mathrm{H}_{2} \mathrm{O}, 1.52 \mathrm{~g} \mathrm{KH}_{2} \mathrm{PO}_{4}, 10 \mathrm{~g}$ sucrose, $2 \mathrm{~g}$ tryptone, $1 \mathrm{~g}$ yeast extract, $1 \mathrm{~g}$ casein, and $2 \mathrm{~mL}$ trace elements [100 mL trace elements contains $2.2 \mathrm{~g} \mathrm{ZnSO}_{4} \cdot 7 \mathrm{H}_{2} \mathrm{O}, 1.10 \mathrm{~g} \mathrm{H}_{3} \mathrm{BO}_{3}, 0.5 \mathrm{~g}$ $\mathrm{MnCl}_{2} \cdot 4 \mathrm{H}_{2} \mathrm{O}, 0.5 \mathrm{~g} \mathrm{FeSO}_{4} \cdot 7 \mathrm{H}_{2} \mathrm{O}, 0.16 \mathrm{~g} \mathrm{CoCl} \cdot 6 \mathrm{H}_{2} \mathrm{O}, 0.16 \mathrm{~g} \mathrm{CuSO}_{4} \cdot 5 \mathrm{H}_{2} \mathrm{O}, 0.11 \mathrm{~g}\left(\mathrm{NH}_{4}\right)_{6} \mathrm{Mo}_{7} \mathrm{O}_{24} \cdot 4 \mathrm{H}_{2} \mathrm{O}$, $5 \mathrm{~g} \mathrm{Na}_{2}$-EDTA]\}. The culture was grown in the dark at $28^{\circ} \mathrm{C}$ on a 150 -rpm shaker for $48 \mathrm{~h}$. The my- 
celial mat was then collected onto sterile filter papers, washed three times with sterile and distilled water, and divided into two equal pieces. One piece was placed in nitrogen-limiting media $(0.52 \mathrm{~g}$ $\mathrm{KCl}, 0.52 \mathrm{~g} \mathrm{MgSO}_{4} \cdot 7 \mathrm{H}_{2} \mathrm{O}, 1.52 \mathrm{~g} \mathrm{KH}_{2} \mathrm{PO}_{4}, 10 \mathrm{~g}$ sucrose, and $2 \mathrm{~mL}$ trace elements), and the other was placed in $1 / 10$ nitrogen media $\left(0.6 \mathrm{~g} \mathrm{NaNO}_{3}, 0.52 \mathrm{~g} \mathrm{KCl}, 0.52 \mathrm{~g} \mathrm{MgSO}_{4} \cdot 7 \mathrm{H}_{2} \mathrm{O}, 1.52 \mathrm{~g} \mathrm{KH}_{2} \mathrm{PO}_{4}\right.$, $10 \mathrm{~g}$ sucrose, and $2 \mathrm{~mL}$ trace elements). The two cultures were grown in the dark at $28^{\circ} \mathrm{C}$ on a 150-rpm shaker. The mycelia mats from each treatment were collected after $24 \mathrm{~h}$ and $48 \mathrm{~h}$, washed with sterile and distilled water, and RNA extraction was performed. Three independent biological samples were used with specific primers for each individual gene.

\section{Inoculation of rice leaves with spores}

Fungal conidia were obtained by scraping 10 -day-old cultures grown at $28^{\circ} \mathrm{C}$. Mycelia were separated from conidia by filtration through two layers of Miracloth. The concentration was adjusted to $2 \times 10^{5}$ spores $/ \mathrm{mL}$ using sterile distilled water. For spore inoculation, spore suspensions were sprayed on to the surface of leaves harvested from three-leaf-stage and whole-plant seedlings, and rice whole-plant seedlings inoculated with strains were placed in an incubator (with high humidity) at $28^{\circ} \mathrm{C}$. The leaves from $M$. oryzaestrain-inoculated wholeplant seedlings were collected at $0,24,48,72,96$, and $168 \mathrm{~h}$ post-inoculation (hpi), and immediately frozen using liquid nitrogen and ground using TRIzol reagent (Invitrogen; Carlsbad, CA, USA). Three independent biological samples were used with specific primers for each individual gene. The data were normalized using the actin genes as an internal control, foldchange expression level was calculated by comparing with healthy rice leaves at $0 \mathrm{hpi}$, and standard deviations were calculated.

\section{RNA extraction and cDNA synthesis}

Total RNA was extracted from frozen fungal tissues, and from infected and mock-inoculated rice leaves using TRIzol reagent (Invitrogen) according to the manufacturer protocol. Total RNA was purified using the RNeasy Plant Mini Kit (Invitrogen) according to the manufacturer protocol, and then used for reverse transcription. First-strand cDNA was synthesized using $2 \mu \mathrm{g}$ total RNA template, Oligo (dT) primer, and M-MLV reverse transcriptase (Invitrogen) according to the manufacturer protocol.

\section{Real-time RT-PCR}

To assess the expression of genes encoding small secreted proteins under conditions of $1 / 10$ nitrogen, nitrogen starvation, and during rice infection, the relative expression levels of seven genes encoding MGS00074, MGS0274, MGS0338, MGS0718, MGS0997, MGS1242, and MGS1460 were determined using real-time PCR. The level of actin gene amplification was used as a positive control. Data were analyzed using the delta delta-CT method. The real-time RT-PCR was conducted using $\mathrm{iQ}^{\mathrm{TM}} \mathrm{SYBR}^{\circledR}$ Green Supermix (Bio-Rad Laboratories, Inc., USA). The conditions were as follows for a $25-\mu \mathrm{L}$ reaction: $12.5 \mu \mathrm{L}$ qPCRSupermix, $0.5 \mu \mathrm{L}$ of each $10 \mu \mathrm{M}$ forward and reverse primers, and $2 \mu \mathrm{L}$ cDNA template. Primer sequences are listed in Table 1. 
Table 1. Primer pairs used in real-time RT-PCR.

\begin{tabular}{|c|c|}
\hline Gene & Primer sequence \\
\hline MG03982.5 & F: 5'-GTCGCTCTTGACTTTGAGCA-3' \\
\hline (actin) & R: 5'-ATACCACCGCTCTCAAGACC-3' \\
\hline \multirow[t]{2}{*}{ MGS0074 } & F: 5'-TCTCGGGGATGATTCAATCT-3' \\
\hline & R: 5'-CAGTTAGCGGTGTATTTCTC-3' \\
\hline \multirow[t]{2}{*}{ MGS0274 } & F: 5'-GATCCATGCGTCACAATCAG-3' \\
\hline & R: 5'-GAGATGAATAACCGCGGACA-3' \\
\hline \multirow[t]{2}{*}{ MGS0338 } & F: 5'-GTCAACGAGGCGCATCTACT-3' \\
\hline & R: 5'-GCACTCGCCATTCTGGATCT-3' \\
\hline \multirow[t]{2}{*}{ MGS0718 } & F: 5'-CCGTGACCCGATGATAATTC-3' \\
\hline & R: 5'-CTGAGGCAGATAGGGCATTT-3' \\
\hline \multirow[t]{2}{*}{ MGS0997 } & F: 5'-CCCAGGAAACCTGCATGA-3' \\
\hline & R: 5'-TTAGGCCAGTTGTTGCGG-3' \\
\hline \multirow[t]{2}{*}{ MGS1242 } & F: 5'-ACTCAAGAGCCCCAGCAAC-3' \\
\hline & R: 5'-TCCACGAAGGCTTGTTGTC-3' \\
\hline \multirow[t]{2}{*}{ MGS1460 } & F: 5'-GTGCCGCCTCAATATACGAC-3' \\
\hline & R: 5'-TCTACCGCCTTGTGAGGACT-3' \\
\hline
\end{tabular}

\section{RESULTS}

\section{Sequence characterization of secreted proteins in $M$. oryzae}

Signal peptides of candidate protein sequences containing fewer than 100 aa in M. oryzaewere predicted using internet-based software such as SignalP4.0, TargetP V1.1, Big-PI predictor, and TMHMM v2.0. The predicted results showed that MGS0074, MGS0274, MGS0338, MGS0718, MGS0997, MGS1242, and MGS1460 contain a signal peptide, no transmembrane helix, no GPIanchor site, and are transported via the Sec pathway. Based on these data, we predicted that these seven proteins were putative secretory proteins without any homologous genes identifiable in the $\mathrm{NCBI}$ database. The molecular weight of the seven proteins was predicted to be less than $10 \mathrm{kDa}$. Therefore, these proteins were identified as small secretory proteins.

We analyzed the number of cysteine residues present in the seven protein sequences. MGS0338, MGS0718, MGS0997, and MGS1460 each contained four cysteine residues, MGS0074 and MGS0274 contained six cysteine residues, and MGS1242 contained nine cysteine residues. The promoters of each of the seven genes contained GATA sequences. Subcellular location of these small cysteine-rich proteins was predicted using MultiLoc/TargetLoc, which revealed that all seven proteins are extracellular. In addition, the hydrophobic characteristics of the seven proteins were analyzed, and all proteins except MGS1242 were hydrophobic.

\section{Gene expression in different strains cultured in complete media}

In order to detect the expression of MGS0074, MGS0274, MGS0338, MGS0718, MGS0997, MGS1242, and MGS1460, which encode secretory proteins, in two strong pathogenic strains (Y98-16 and 95-23-4a) and two weak pathogenic strains (Y99-63 and 94-64-1b) cultured in complete media, real-time RT-PCR was used. Gene expression in the weak pathogenic strain, Y99-63, was set to 1, and there was reduced expression of MGS0074, MGS0274, MGS0718, and MGS1242 in the strong pathogenic strain Y98-16, and MGS0074, MGS0274, MGS0718, MGS0997, and MGS1242 in the strong pathogenic strain, 95-23-4a. MGS0338 and MGS1460 were differently upregulated in both Y98-16 and 95-23-4a. All seven genes were upregulated more than 2-fold in the weak pathogenic strain 94-64-1b (Figure 1). MGS0338 and MGS1460 were up- 
regulated in Y98-16, 95-23-4a, and 94-64-1b more than 2-fold, and their expression level was very similar in all three strains (Figure 1). Therefore, among the seven genes, the expression of three was repressed in strong pathogenic strains, all seven were upregulated in the weak pathogenic strain, and two were upregulated in both the weak and strong pathogenic strains.

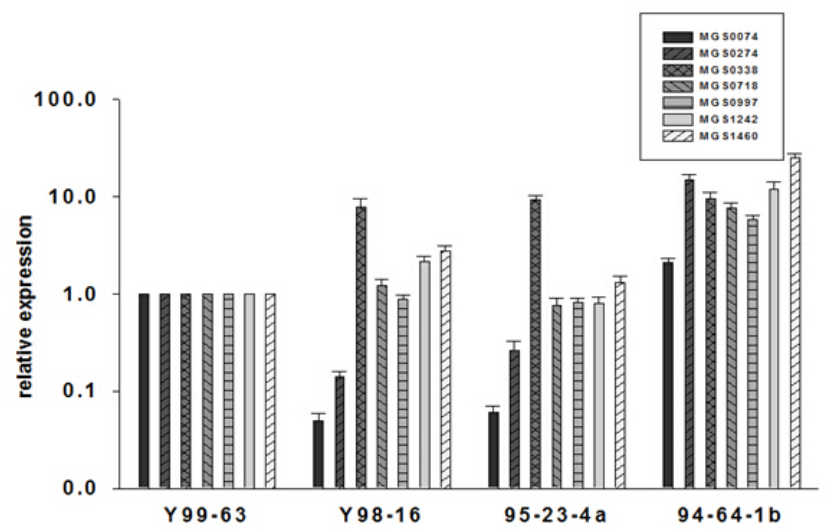

Figure 1. Expression level of seven genes in different strains cultured in complete media. Expression data in the strains Y98-16, 95-23-4a, and 94-64-1b cultured in complete media are provided relative to Y99-63. Error bars represent mean \pm SD. Essentially identical results were obtained in three independent experiments.

\section{Expression of genes in Y98-16 and Y99-63 cultured in 1/10-N media and nitrogen starvation media}

To compare gene expression in the strong pathogenic strain, Y98-16, and the weak pathogenic strain, Y99-63, during culture with 1/10-N, nitrogen starvation, and complete media, we determined the expression of MGS1242 and MGS1460 in both strains at 24 and 48 h. MGS1242 was significantly upregulated in the weak pathogenic strain Y99-63 when cultured in 1/10-N media and under nitrogen starvation for $24 \mathrm{~h}$ and $48 \mathrm{~h}$, whereas the expression MGS1460 was depressed (Figure 2a and b). MGS1242 was also significantly upregulated more than 2-fold in the strong pathogenic strain of Y98-16 when cultured in 1/10-N media and under nitrogen starvation for $24 \mathrm{~h}$ and $48 \mathrm{~h}$; expression of MGS1460 was also depressed (Figure 2c and d). These data show that a higher level of MGS1242 expression was observed when cultured in both $1 / 10 \mathrm{~N}$ media and under nitrogen starvation for 24 and $48 \mathrm{~h}$, which indicates that increased expression of this gene was not relative to the amount of nitrogen limitation since higher levels gene expression were observed under nitrogen limitation.

\section{Expression of genes encoding small secreted proteins during rice blast fungus infection}

The expression levels of MGS0074, MGS0274, MGS0338, MGS0718, MGS0997, MGS1242, and MGS1460 in Y99-63 were analyzed using real-time PCR. These seven genes were expressed at higher levels at 24 and $48 \mathrm{hpi}$, with maximum expression being observed at 48 hpi (Figure 3). With the exception of MGS00074, the expression of these genes was upregulated more than 2-fold from 24 to $96 \mathrm{hpi}$, there was reduced expression of MGS0074 and MGS1460 at $168 \mathrm{hpi}$, whereas the other five genes still displayed more than 2-fold expression level (Figure 3). These data show that most of the secreted protein in $M$. oryzae is involved in plant infection. 

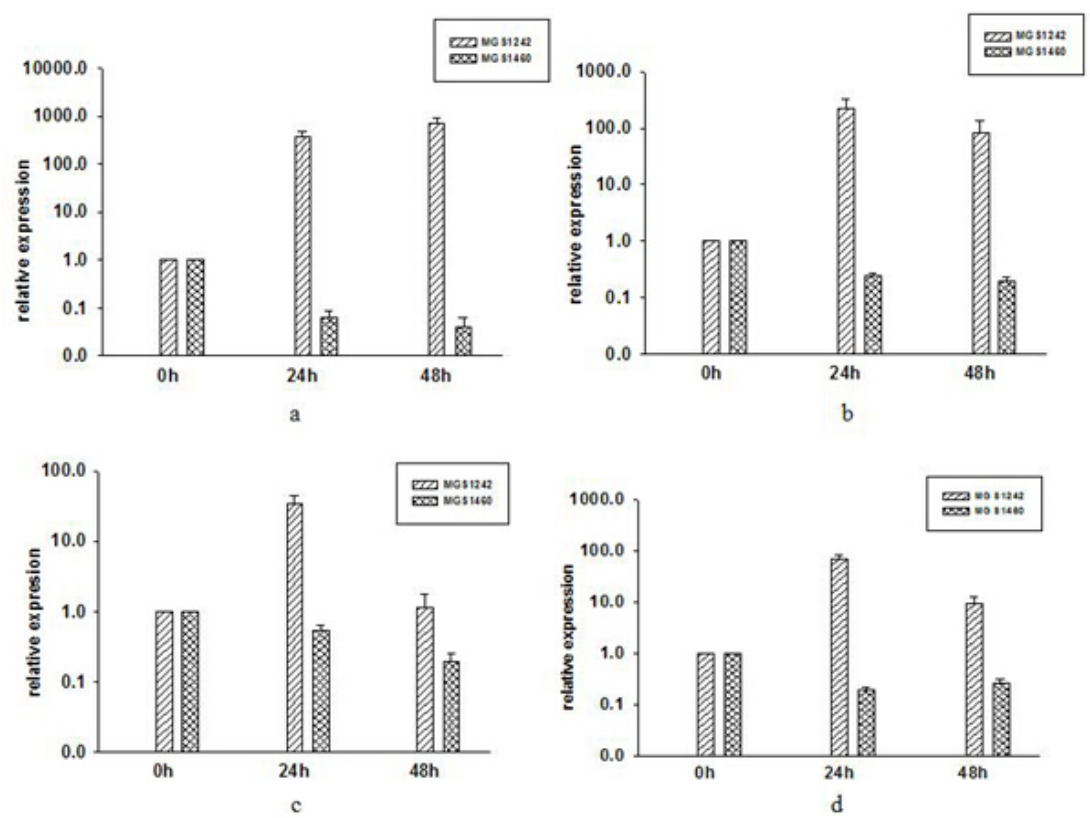

Figure 2. Expression level of NUT1, MGS1242, and MGS1460 in Y98-16 and Y99-63 cultured in 1/10-nitrogen media and nitrogen-starvation media for 24 and $48 \mathrm{~h}$. Relative expression levels of genes in the weak pathogenic strain Y9963 cultured in 1/10-nitrogen media (a) and nitrogen-starvation media (b). Relative expression of genes in the strong pathogenic strain Y98-16 cultured in 1/10-nitrogen media (c) and nitrogen-starvation media (d). Expression data in two strains cultured in 1/10-nitrogen media and nitrogen-starvation media for $24 \mathrm{~h}$ are provided relative to Actin. Error bars representmean $\pm \mathrm{SD}$. Essentially identical results were obtained in three independent experiments.

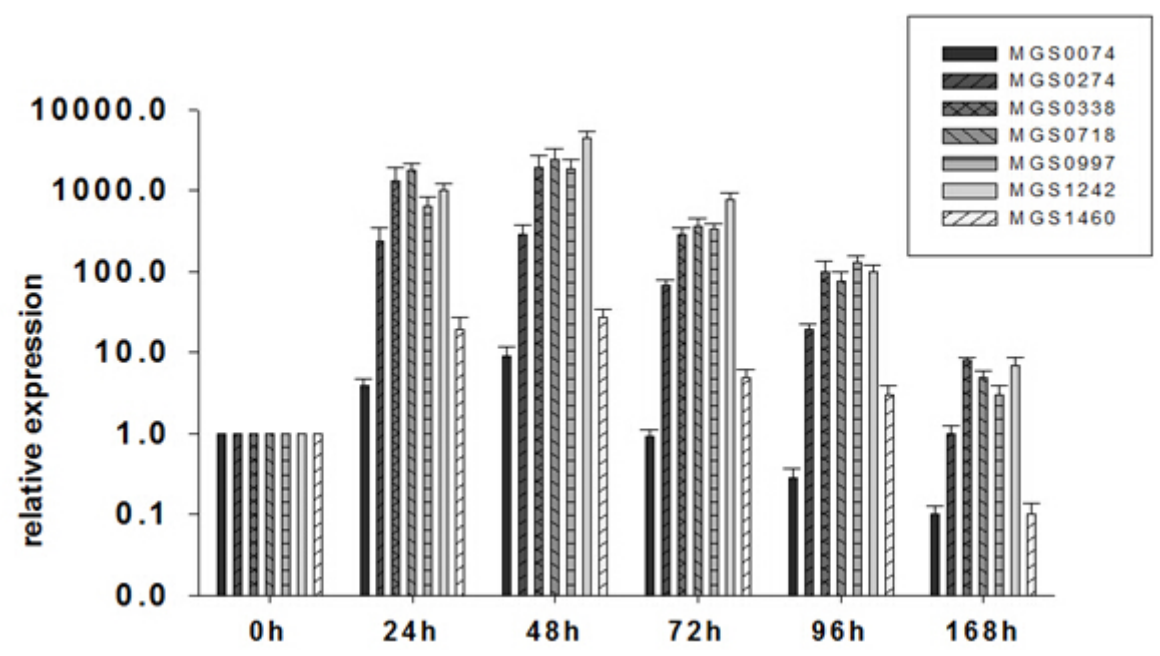

Figure 3. Expression level of seven genes at different stages post inoculation during Y99-63 infection of the rice cultivar Lijiangxintuanheigu. Expression data at $0,24,48,72,96$, and $168 \mathrm{~h}$ post-inoculation (hpi) are provided relative to Actin. Error bars represent mean \pm SD. Essentially identical results were obtained in three independent experiments 


\section{DISCUSSION}

Several fungal genomes have been sequenced in order to identify secreted proteins and to study their roles in the interaction between fungi and host plants (Martin et al., 2011). Secreted proteins are translocated into host tissues during pathogen infection and function in the extracellular space or in the cytoplasm (Stergiopoulos et al., 2009; de Joung et al., 2011). Secreted proteins play an important role during blast fungus infection in rice; however, the functional mechanism and mode of action have not been well studied (Valent et al., 2010; Mentlak et al., 2012). It is important to delineate the intrinsic functions of different pathogen-secreted proteins in order to uncover the mechanism of rice interaction with blast fungus. The seven candidate genes described herein, which encode small secreted proteins, were obtained by analyzing the blast genome using combination software. These secreted proteins are small, contain an N-terminal signal peptide, are rich in cysteine residues, and most have a hydrophobic character. This analysis provides a basis for the subsequent cloning of these genes.

One environmental factor that can induce the expression of pathogenicity related genes is nitrogen limitation, which is of great significance in disease development (Joosten et al., 1994). Talbot et al. (1997) found that several genes were induced in blast strains cultured in nitrogen starvation media, and that these genes were also expressed during rice infection by blast fungus. These authors also reported that nitrogen limitation can induce the expression of some senescencerelated genes. Donofrio et al. (2006) found that nitrogen limitation induced the expression of genes that were also detected during the interaction between pathogens and host plants. Several genes were found to be upregulated during plant infection and nitrogen starvation (Coleman et al., 1997; Stephenson et al., 1997; Divon et al., 2005; Donofrio et al., 2006). Compatible interactions between a host plant and a pathogen result in the appearance of disease symptoms on the host plant, whereas an incompatible interaction between the two triggers a hypersensitive response in the host (Hogenhout et al., 2009). In the present study, the expression of genes encoding secreted proteins was analyzed when blast strains were cultured in complete media, under nitrogen limitation, and during plant infection. Our results showed that the expression of some genes was repressed in strong pathogenic strains cultured in complete media, but were upregulated in weak pathogenic strains when cultured in nitrogen limitation media. For example, the expression of MGS1242 was repressed in a strong pathogenic strain cultured in complete media, but was expressed at higher levels in both strong and weak pathogenic strains when cultured under nitrogen limitation, which suggests that increased expression of genes encoding secreted proteins was induced by nitrogen limitation, and nitrogen limitation is known to induce the expression of most pathogenicity-related genes. To verify whether the expression of MGS1242 was also increased during plant infection, we analyzed its expression level and the result showed that expression of this gene was higher during plant infection, which indicates that this gene may be involve in pathogenicity. Therefore, from these data, the gene with the highest expression under both nitrogen limitation and plant infection was selected as one that encodes a potential effector protein that can be used for further functional study. Thus, MGS1242 may be considered a potential regulator and co-factor for use when studying the interaction between blast strains and rice. We also detected upregulated gene expression during the stages of rice infection and also under conditions of nitrogen starvation. In addition, all of the seven genes were rich in cysteine residues, and some research has showed that small cysteine-rich proteins play a role in the specificity and pathogenicity of fungal pathogens (Templeton et al., 1994). Thus, the increased expression of these seven genes encoding small cysteine-rich proteins under nitrogen limitation and plant infection might be good candidates for use in subsequent 
functional studies during the interaction of rice with blast fungus. The objective of this study was to identify some candidates that could be used for functional study by investigating the expression level of the putative genes encoding secreted proteins in nitrogen limitation and plant infection while not verify whether the gene deletion or not influence pathogenicity/virulence of $M$. oryzae.

Hydrophobins may play a critical role in pathogenesis and might be involved in establishing basic compatibility between the pathogen and its host (Heath et al., 1981). Cysteine-rich proteins may change the hydrophobicity of the invading mycelia (Hazen et al., 1988), and may play a role in the specificity and pathogenicity of fungal pathogens (Templeton et al., 1994). Our data suggest that the genes encoding small cysteine-rich proteins that are more highly expression under nitrogen limitation and during plant infection may be selected as candidates to enable their function to be studied, but are also highly sensitive to the nitrogen source. Therefore, cysteine-rich proteins secreted by the pathogen may be involved in sensing nitrogen during the interaction between the host and pathogen, especially during the early stage of infection

\section{Conflict of interest}

The authors declare no conflict of interest.

\section{ACKNOWLEDGMENTS}

Research project \#31400073 supported by National Natural Science Foundation of China, and the Yunnan Natural Science foundation (Grant \#2013FB039) from the Yunnan Science and Technology Department of China.

\section{REFERENCES}

Coleman M, Henricot B, Arnau J, and Oliver RP (1997). Starvation-induced genes of the tomato pathogen Cladosporium fulvum are also induced during growth in planta. Mol. Plant Microbe Interact. 10: 1106-1109.

Divon HH, Rothan-Denoyes B, Davydov O, Di Pietro A, et al. (2005). Nitrogen-responsive genes are differentially regulated in planta during Fusariumoxy sporum f. sp. lycopersici infection. Mol. Plant Pathol. 6: 459-470.

Donofrio NM, Oh Y, Lundy R, Pan H, et al. (2006).Global gene expression during nitrogen starvation in the rice blast fungus, Magnaporthe grisea. Fungal Genet. Biol. 43: 605-617.

Hazen BW and Hazen KC. (1988). Dynamic expression of cell surface hydrophobicity during initial yeast cell growth and before germ tube formation of Candida albicans. Infect Immun. 56: 2521-2525.

Heath MC (1981). A generalized concept of host-parasite specificity. Phytopathology 71: 1121-1123.

Hogenhout SA, Van der Hoorn RAL, Terauchi R, and Kamoun S. (2009). Emerging concepts in effector biology of plantassociated organisms. Mol. Plant Microbe Interact. 2: 115-122.

Joosten MH, CozijnsenTJ and De Wit PJ (1994). Host resistance to a fungal tomato pathogen lost by a single base pair change in an avirulence gene. Nature 367: 384-386.

Kamoun S, Klucher KM, Coffey MD and Tyler BM (1993). A gene encoding a host-specific elicitor protein of Phytophthora parasitica. Mol. Plant Microbe Interact. 6: 573-581.

Lau G and Hamer JE (1996). Regulatory genes controlling MPG1 expression and pathogenicity in the rice blast fungus Magnaporthe grisea. Plant Cell 8: 771-781.

Lee SA, Wormsley S, Komoun S, Lee AF, et al. (2003). An analysis of the Candida albicans genome database for soluble secreted proteins using computer-based prediction algorithms. Yeast 20: 595-610.

Magasanik B. (1996). Regulation of gene expression in Escherichia coli. In: Regulation of nitrogen utilization (Lin ECC and Lynch AS, eds.). Cold Spring Harbor Laboratory Press, Cold Spring Harbor, 1344-1356.

Martin F and Kamoun S (2011). Effectors in Plant-Microbe Interactions. John Wiley \& Sons, Inc.

Marzluf GA (1997). Genetic regulation of nitrogen metabolism in the fungi. Microbiol. Mol. Biol. Rev. 62: 17-32.

Mentlak TA, Kombrink A, Shinya T, Ryder LS, et al. (2012). Effector-mediated suppression of chitin-triggered immunity by Magnaporthe oryzae is necessary for rice blast disease. Plant Cell 24: 322335. 
Nespoulous C, Huet JC and Pernoller JC (1992). Structure-function relationship of $\alpha$ and $\beta$ elicitins, signal proteins involved in the plant-Phytophthora interaction. Planta 186: 551-557.

Pernollet JC (1994). Elicitins, ally themselves with plants against parasites. La Recherche 261: 82-84.

Ricci P, Bonnet P, Huet JC, Sallantin M, et al. (1989). Structure and activity of proteins from pathogenic fungi Phytophthora eliciting necrosis and acquired resistance in tobacco and other plant species. Eur. J. Biochem. 183: 555-563.

Snoeijers SS, Vossen P, Goosen T, Van den Broek HW, et al. (1999). Transcription of the avirulence gene Avr9 of the fungal tomato pathogen Cladosporium fulvum is regulated by a GATA-type transcription factor in Aspergillus nidulans. Mol. Gen. Genet. 261: 653-659.

Snoeijers SS, Pérez-García A, Joosten MHAJ, De Wit PJGM (2000). The effect of nitrogen on disease development and gene expression in bacterial and fungal plant pathogens. Eur. J. Plant Pathol. 106: 493-506.

Stephenson SA, Green JR, Manners JM and Maclean DJ (1997). Cloning and characterisation of glutamine synthetase from Colletotrichumgloeosporioides and demonstration of elevated expression during pathogenesis on Stylosanthes guianensis. Curr. Genet. 31: 447-454.

Stergiopoulos I and de Wit PJ (2009). Fungal effector proteins. Annu. Rev. Phytopathol. 47: 233-263.

Talbot NJ, Ebbole DJ and Hamer JE (1993). Identification and characterization of Mpg1, a gene involved in pathogenicity from the rice blast fungus Magnaporthe grisea. Plant Cell 5: 1575-1590.

Talbot NJ, McCafferty HRK, Ma M, Moore K, et al. (1997). Nitrogen starvation of the rice blast fungus Magnaporthe grisea may act as an environmental cue for disease symptom expression. Physiol. Mol. Plant. Pathol. 50: 179-195.

Templeton MD, Rikkerink EHA and Beever RE (1994). Small, cysteine-rich proteins and recognition in fungal-plant interactions. Mol. Plant. Microbe Interact. 7: 320-325.

Valent B and Khang CH (2010). Recent advances in rice blast effector research. Curr. Opin. Plant. Biol. 13: 434-441.

Van den Ackerveken GF, Dunn RM, Cozijnsen AJ, Vossen JP, et al. (1994). Nitrogen limitation induces expression of the avirulence gene Avr9 in the tomato pathogen Cladosporium fulvum. Mol. Gen. Genet. 243: 277-285.

Wevelsiep L, Kogel KH and Knogge W. (1991). Purification and characterization of peptides from Rhynchosporium secalis inducing necrosis in barley. Physiol. Mol. Plant. Pathol. 39: 471-482. 